\title{
Interstitial Nephritis Associated with Glomerulonephritis in a Patient with Hashimoto's Disease and Idiopathic Portal Hypertension
}

\author{
Hidetsugu Sasaki, Kensuke Joh*, Isako Ohtsuka, Hideki Ohta, Tsutomu OHhashi, Seiji Hoashi, \\ Takamune TAKaHashi*, Tadaaki ToKudA*, Katsuichi Koyama and Yukihide IsogaI
}

\begin{abstract}
A middle-aged woman with hypothyroidism, idiopathic portal hypertension and nephrotic syndrome is presented. This unusual clinical appearance could not be explained as SLE by serological examinations. Pathohistological examinations showed "Banti's liver", Hashimoto's thyroiditis and diffuse proliferative glomerulonephritis with severe tubulo-interstitial nephritis. Immunohistochemical studies revealed IgA deposits in glomeruli. Electron microscopic study disclosed peculiar lucent areas of rarefaction with osmiophilic particles in tubular basement membranes. This tubulointerstitial nephritis was considered to be related to the immunological mechanism involving thyroid gland, liver and kidney disorders. This case thus had a clinically rare combination of these three.
\end{abstract}

(Internal Medicine 31: 641-648, 1992)

Key words: nephrotic syndrome, IgA deposits, osmiophilic body, hepatic glomerulosclerosis, hypergammaglobulinemia

\section{Introduction}

Major forms of glomerulopathies in association with thyroid disorders (Grave's disease and Hashimoto's disease) or hepatic disorders (cirrhosis of liver and chronic hepatitis) have been reported to be membranous (1) and diffuse proliferative glomerulonephritis $(2,3)$. Interstitial nephritis, however, had not been demonstrated in the cases associated with thyroidal and hepatic disorders reported previously.

In this report, we describe a female patient with hypothyroidism with the two additional clinical conditions of idiopathic portal hypertension and nephrotic syndrome. We confirmed that the patient had Hashimoto's disease, "Banti's liver and spleen", as well as IgA deposits coexisting with severe tubulo-interstitial nephritis. As far as we know, this is the first report of a peculiar osmiophilic body found in the tubular basement membrane associated with severe interstitial nephritis. The presence of several autoantibodies suggests that a rare combination of the diseases is based on immune-mediated mechanisms.

\section{Case Report}

The patient, a 46-year-old female, visited her family doctor complaining of shortness of breath in January 1986, and was diagnosed as having anemia. In March, leukopenia and thrombocytopenia developed. Her past medical history included chronic sinusitis at the age of 20 . There was no history of jaundice, edema, arthralgia or tonsillitis. There was also no history of blood transfusions. She did not smoke or drink alcohol. There was no family history of thyroid disorders.

Physical examination (May 24, 1986) revealed that her blood pressure was $142 / 86 \mathrm{mmHg}$, her pulse rate was $80 / \mathrm{min}$, her respiration rate was $16 / \mathrm{min}$ and her temperature was $36.7^{\circ} \mathrm{C}$. She was well-nourished, not icteric, and had no cervical or axillary lymphadenopathy. Her lungs and heart were normal. Abdominal examination showed splenomegaly, with the spleen being palpable four finger breadths below the left costal margin, and no hepatomegaly. Physical findings included a non-tender and elastic hard goiter. There was no pitting edema of her legs and no vascular spider or palmar erythema.

From the Third Department of Internal Medicine and * the Department of Pathology, Jikei University School of Medicine, Tokyo Received for publication April 30, 1991; Accepted for publication December 27, 1991

Reprint requests should be addressed to Dr. Hidetsugu Sasaki, the Daisan Hospital, Jikei University School of Medicine, 4-11-1, Izumihoncho, Komae, 201, Japan 
Table 1. Laboratory Data on First Admission

(May 24, 1986)

\begin{tabular}{|c|c|c|c|c|c|}
\hline \multicolumn{4}{|l|}{ Blood } & \multicolumn{2}{|l|}{ Serology } \\
\hline $\mathrm{Ht}$ & $36.6(\%)$ & Alp & $100(\mathrm{mU} / \mathrm{ml})$ & Immunoglobulin & \\
\hline $\mathrm{Hg}$ & $12.6(\mathrm{~g} / \mathrm{dl})$ & $\mathrm{T}$. protein & $7.3(\mathrm{~g} / \mathrm{dl})$ & $\operatorname{IgG}$ & $2,611(\mathrm{mg} / \mathrm{dl})$ \\
\hline $\mathrm{RBC}$ & $417 \times 10^{4}\left(/ \mathrm{mm}^{3}\right)$ & Albumin & $3.4(\mathrm{mg} / \mathrm{dl})$ & $\operatorname{IgA}$ & $336(\mathrm{mg} / \mathrm{dl})$ \\
\hline WBC & $2,000\left(/ \mathrm{mm}^{3}\right)$ & Proteinphoresis & & $\operatorname{IgM}$ & $73(\mathrm{mg} / \mathrm{dl})$ \\
\hline Plt & $5.3 \times 10^{4}\left(/ \mathrm{mm}^{3}\right)$ & Albumin & $55.5(\%)$ & Anti-thyroglobulin antibody & - \\
\hline Urine & & $\alpha_{1}$-globulin & $2.2(\%)$ & Anti-microsome antibody & $+(256,000 \times)$ \\
\hline Protein & $2+$ & $\alpha_{2}$-globulin & $5.6(\%)$ & AMA & - \\
\hline Sugar & - & $\beta$-globulin & $5.8(\%)$ & ASMA & $+(160 \times)$ \\
\hline S.G. & 1.024 & $\gamma$-globulin & $30.9(\%)$ & RA factor & - \\
\hline Sediments & & BUN & $18(\mathrm{mg} / \mathrm{dl})$ & ANA & $+(80 \times)$ \\
\hline RBC: $10-15 / \mathrm{HPF}$ & & Creatinine & $0.8(\mathrm{mg} / \mathrm{dl})$ & Anti-DNA antibody & $4.9(\mathrm{U} / \mathrm{ml})$ \\
\hline WBC: $1-2 / \mathrm{HPF}$ & & Thyroid function & & Complements & \\
\hline Blood chemistry & & TSH & $180(\mu \mathrm{U} / \mathrm{ml})$ & $\mathrm{C} 3$ & $48(\mathrm{mg} / \mathrm{dl})$ \\
\hline T. bilirubin & $0.7(\mathrm{mg} / \mathrm{dl})$ & $\mathrm{T}_{3}$ & $0.5(\mathrm{ng} / \mathrm{ml})$ & $\mathrm{C} 4$ & $9(\mathrm{mg} / \mathrm{dl})$ \\
\hline D. bilirubin & $0.2(\mathrm{mg} / \mathrm{dl})$ & $T_{4}$ & $3.2(\mu \mathrm{g} / \mathrm{dl})$ & $\mathrm{CH}_{50}$ & $30.6(\mathrm{U} / \mathrm{ml})$ \\
\hline $\mathrm{LDH}$ & $178(\mathrm{mU} / \mathrm{mI})$ & Renal funcution & & LE factor & - \\
\hline GOT & $30(\mathrm{mU} / \mathrm{ml})$ & Clcreat & $90.0(\mathrm{ml} / \mathrm{min})$ & $\mathrm{HB}_{\mathrm{s}}$ antigen & - \\
\hline GPT & $19(\mathrm{mU} / \mathrm{ml})$ & & & Anti- $\mathrm{HB}_{\mathrm{s}}$ antibody & - \\
\hline
\end{tabular}

AMA: anti-mitochondrial antibody, ASMA: anti-smooth muscle antibody, ANA: anti-nulear antibody, Clereat: Creatinine clearance.

Laboratory data obtained at first admission are shown in Table 1. Her blood contained normal-appearing mature leukocytes in reduced numbers, and her bone marrow was normocellular. The hemoglobin level was normal, and had been improved by oral ferrous sulfate therapy. Her abnormal findings included thrombocytopenia and evidence of immunological and thyroid disorders.

First, there was hypergammaglobulinemia, which was due to a markedly increased immunoglobulin(Ig) $G$ level. In addition to this, anti-nuclear antibody and antismooth muscle antibody were positive at a low titer, while anti-microsomal antibody was positive at a high titer. The serum complement level was at the lower limit of the normal range. Both hepatitis B surface antigen and anti-HBsAg antibody were negative. The transaminase and alkaline phosphatase level were not evaluated in the liver function studies.

Secondly, thyroid function studies showed low T4 and T3 level by radioimmunoassay, and a high basal level of thyroid-stimulating hormone. Urinalysis disclosed microhematuria and proteinuria, but casts were absent. Neither hypoproteinemia nor elevation of the serum creatinine level was present.

Barium examination of the upper gastrointestinal tract showed the presence of varices in the lower part of the esophagus. Endoscopy detected the presence of three erythematous varices in the middle portion of the esophagus. Abdominal ultrasonography demonstrated massive splenomegaly, with marked dilatation of extrahepatic collateral veins and collateral flow via dilatated veins in the retroperitoneal space (Fig. 1). Abdominal CT scan revealed massive splenomegaly.

The presence of splenomegaly, thrombocytopenia, and esophageal varices suggested hypersplenism with portal hypertension in this patient. Accordingly, sple-

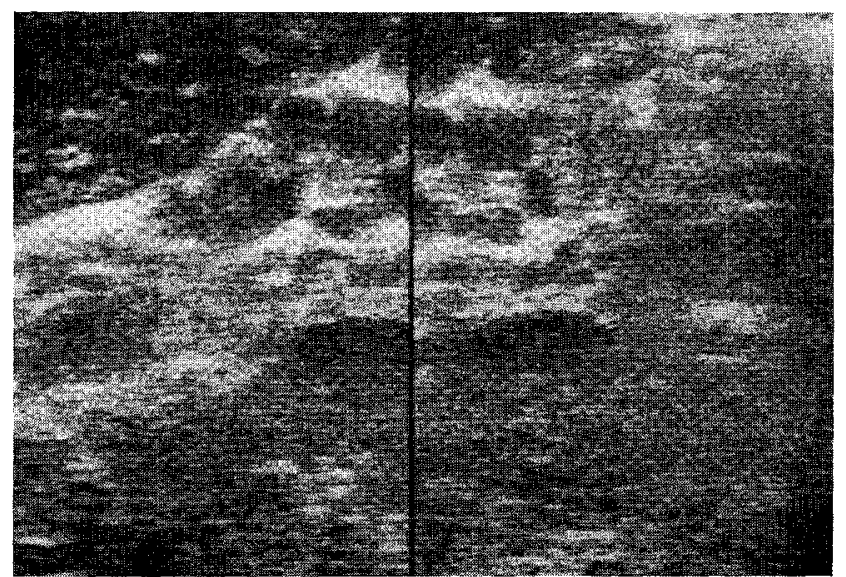

Fig. 1. Ultrasonographic image. The splenic vein is clearly seen and there is marked dilatation of extrahepatic collateral veins.

nectomy was performed after her admission. At operation (June 26, 1986), the spleen was removed (weight $900 \mathrm{~g}$ ) and liver and thyroid biopsies were performed. The liver biopsy specimens showed mild infiltration of lymphocytes into Glisson's sheath which resulted in mild fibrotic extension. However, the division of parenchyma by fibrotic zones remained incomplete. The heterotropic portal branches which were separated from Glisson's sheath as well as from the hepatic vein were seen. This feature is characteristic of the manifestation in the liver of idiopathic portal hypertension called "Banti's liver (4) and spleen (5)" (Fig. 2). The histological appearance of the spleen was typical of the Banti's-spleen characterized by a remarkable sinus hyperplasia.

Open biopsy of the thyroid gland demonstrated severe infiltration of lymphocytes into the parenchyma forming enlarged secondary follicle formation. The thyroid gland 


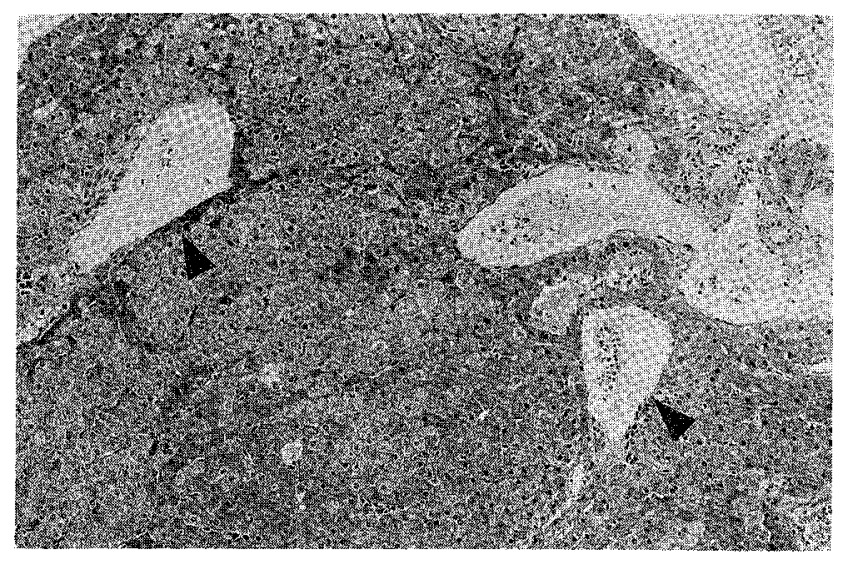

Fig. 2. Liver biopsy specimen. Heterotopic and dilated portal veins are seen in the hepatic lobules (arrows) (H-E stain). Predominant infiltration of lymphocytes is seen in Glisson's sheath.

displayed marked atrophy. The pathological diagnosis of the thyroid gland was Hashimoto's thyroiditis. During her hospital stay, she was administered oral levothyroxine sodium (0.1 mg/day) in July 1986.

Approximately two years later, she developed edema of the lower extremities. Laboratory findings on admission when the renal biopsy was performed in March 1990 are shown in Table 2. Urinalysis revealed severe hematuria with severe proteinuria. Several oval fat bodies were found in the urine, and the 24-hour urinary protein excretion was $2.6 \mathrm{~g}$ up to $6.1 \mathrm{~g}$. Bacteria and WBC casts were not found in fresh urine. The total serum protein level was decreased markedly and electrophoresis showed a pattern of the nephrotic syndrome. Her creatinine clearance level was decreased.
Autoantibodies were studied again. After splenectomy, anti-microsomal antibody had declined to a low titer and the anti-smooth muscle antibody titer had become negative. On the other hand, anti-nuclear antibody remained at the same titer as before operation. At the same time, the anti-single-stranded DNA IgG antibody titer and anti-thyroglobulin antibody were positive. In addition to the above autoantibodies, circulating immune complexes (CICs) were detected by the Clq and Raji cell assays. Anti-RNP antibody and anti-Sm antibody were negative. Anti-SS-A antibody and anti-SS-B antibody were also negative. HCV antibody was negative.

The renal biopsy specimens were taken mainly from the renal cortex and included 48 glomeruli. There were 18 glomeruli with global sclerosis, 12 with adhesion, and 6 with moderate fibrocellular crescents. The other 12 glomeruli showed diffuse mesangial stalk thickening with mesangial hypercellularity. Tubuli showed marked atrophy ( $35 \%$ of the total field) and were surrounded by interstitial fibrosis with severe infiltration of lymphocytes and plasma cells. Afferent arteries exhibited moderate hypertrophy in the media (Fig. 3).

Immunohistochemical studies revealed diffuse and predominant staining for IgA (Fig. 4), IgM in the mesangial area and along the capillaries. $\mathrm{C} 3$ and $\mathrm{Clq}$ showed a focal and segmental pattern in the mesangium. IgG was negative. The tubular basement membrane showed neither immunoglobulin nor complement deposition. Thyroglobulin was also negative in glomeruli.

Electron microscopy showed that the dense deposits were found sporadically and irregularly in the mesangial matrix as well as in the intramembranous area. Proliferated mesangial cells were associated with

Table 2. Laboratory Data on Second Admission

\begin{tabular}{|c|c|c|c|}
\hline & & & (Feb. 5, 1990) \\
\hline \multicolumn{2}{|l|}{ Urine } & \multicolumn{2}{|l|}{ Proteinphoresis } \\
\hline Protein & $2+$ & Alubumin & $43.3(\%)$ \\
\hline Sugar & - & $\alpha_{1}$-globulin & $9.1(\%)$ \\
\hline S. G. & 1.019 & $\alpha_{2}$-globulin & $13.9(\%)$ \\
\hline Sediments & & $\beta$-globulin & $10.3(\%)$ \\
\hline $\mathrm{RBC}$ & many/HPF & $\gamma$-globulin & $23.4(\%)$ \\
\hline WBC & $1-2 / \mathrm{HPF}$ & Serology & \\
\hline Fatty cast & 7/HPF & ANA & $+(160 \times)$ \\
\hline Protein excretion & 4.4 (g/day) & Anti-ss DNA IgG & $35(\mathrm{U} / \mathrm{ml})$ \\
\hline \multicolumn{2}{|l|}{ Blood chemistry } & Anti-ds DNA IgG & $1(\mathrm{U} / \mathrm{ml})$ \\
\hline BUN & $34(\mathrm{mg} / \mathrm{dl})$ & Anti-SS-A antibody & - \\
\hline Creatinine & $1.7(\mathrm{mg} / \mathrm{dl})$ & Anti-SS-B antibody & - \\
\hline Uric acid & $8.5(\mathrm{mg} / \mathrm{dl})$ & Anti-RNP antibody & - \\
\hline \multicolumn{2}{|l|}{ Renal funtion } & Anti-Sm antibody & - \\
\hline Clcreat & $44.3(\mathrm{ml} / \mathrm{min})$ & $\mathrm{HCV}$ antibody & - \\
\hline \multicolumn{2}{|l|}{ Blood chemistry } & Anti-thyroglobulin antibody & $7.1(\mu \mathrm{g} / \mathrm{ml})$ \\
\hline T. protein & $5.4(\mathrm{~g} / \mathrm{dl})$ & Circulating immune complexes & \\
\hline \multirow[t]{3}{*}{ Albumin } & $2.6(\mathrm{~g} / \mathrm{dl})$ & $\mathrm{Clq}$ & $4.3(\mu \mathrm{g} / \mathrm{ml})$ \\
\hline & & $\mathrm{C}^{1}$ & $8(\mu \mathrm{g} / \mathrm{ml})$ \\
\hline & & Raji Cell & $50(\mu \mathrm{g} / \mathrm{ml})$ \\
\hline
\end{tabular}

ANA: anti-nuclear antibody, SS: Sjogren's syndrome, Clereat: creatinine clearance. 


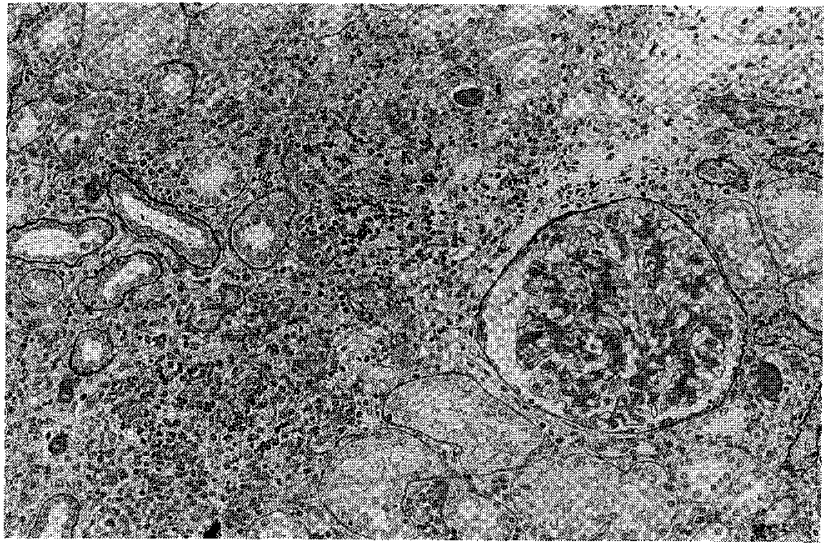

Fig. 3. Renal biopsy specimen. Light micrograph of renal biopsy specimen. Glomerulus shows diffuse mesangial proliferation. Atrophic tubuli are surrounded by interstitial fibrosis with lymphocytic infiltration (PAS stain).

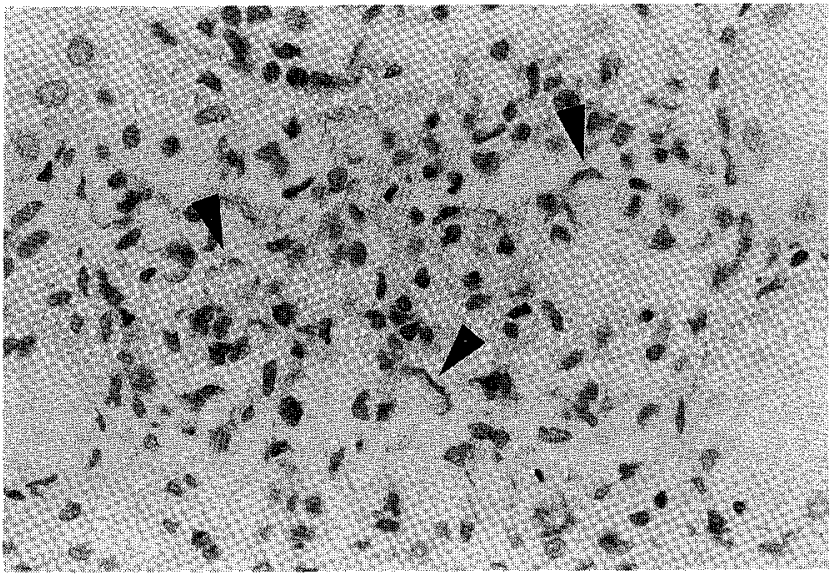

Fig. 4. Renal biopsy specimen. An immunohistochemical study shows that IgA (arrows) is diffusely and globally positive in the paramesangial areas of glomeruli $(\times 400)$.

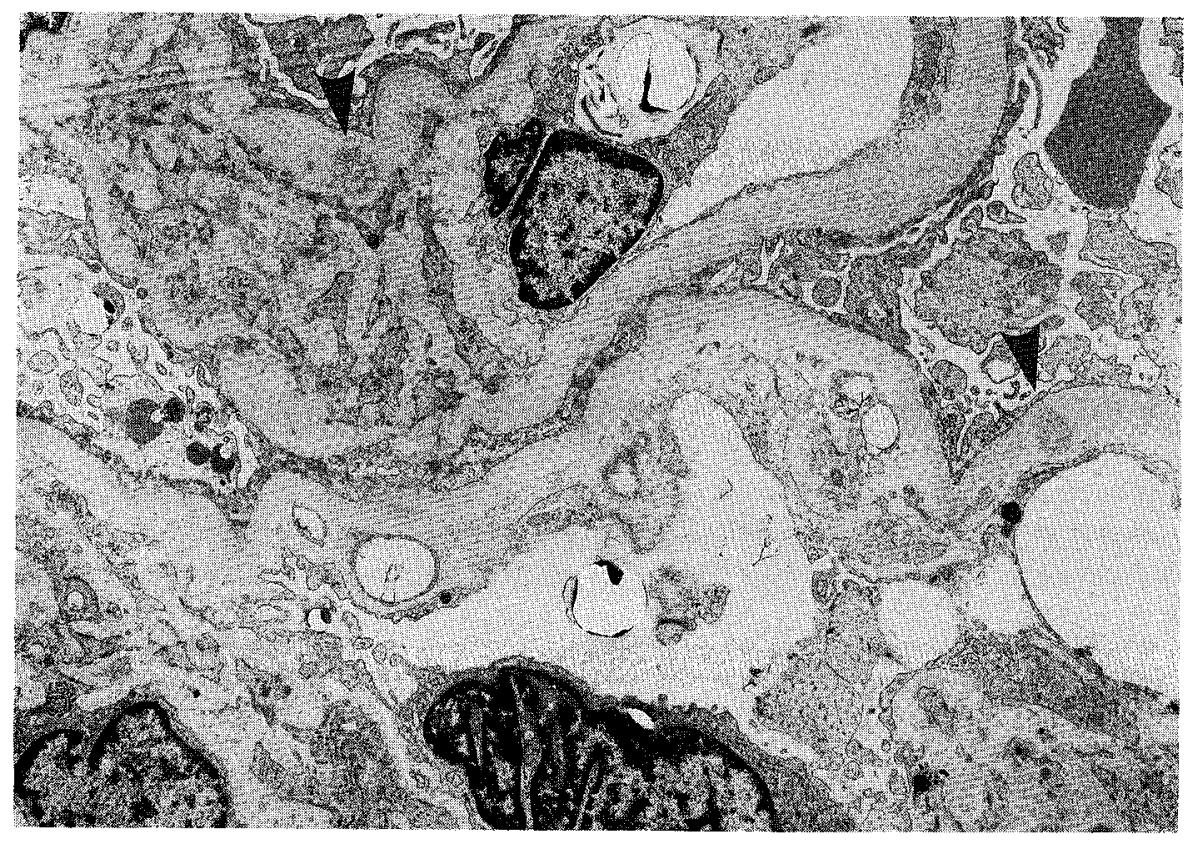

Fig. 5. Renal biopsy specimen. Electron micrograph shows that the dense deposits (arrows) are found sporadically and irregularly in the mesangial matrix as well as in the intramembranous areas $(\times 6,000)$.

local mesangial interposition. The epithelial foot processes were effaced in almost part of capillary loops (Fig. 5). Electron-dense deposits were not found in the tubular basement membrane (TBM), although the tubular basement membrane contained numerous electron lucent areas of irregular rarefaction with and without peculiar osmiophilic particles. They were not limited by the membrane (Fig. $6 \mathrm{~A}$ and $\mathrm{B}$ ). Foam cells were often found in the interstitium.

\section{Discussion}

Here we present a case of a middle-age woman with Hashimoto's disease and idiopathic portal hypertension (IPH). Furthermore, development of nephrotic syndrome could be documented over two years. However, systemic lupus erythematosus (SLE) and Sjögren's syndrome (SS) could not be diagnosed on the basis of absent clinical findings inclusive of arthritis, sicca syndrome, fever, skin eruptions and positive LE cell test and antiSS-A as well as anti-SS-B. It is of considerable interest 


\section{Interstitial Nephritis in Hashimoto's Disease}
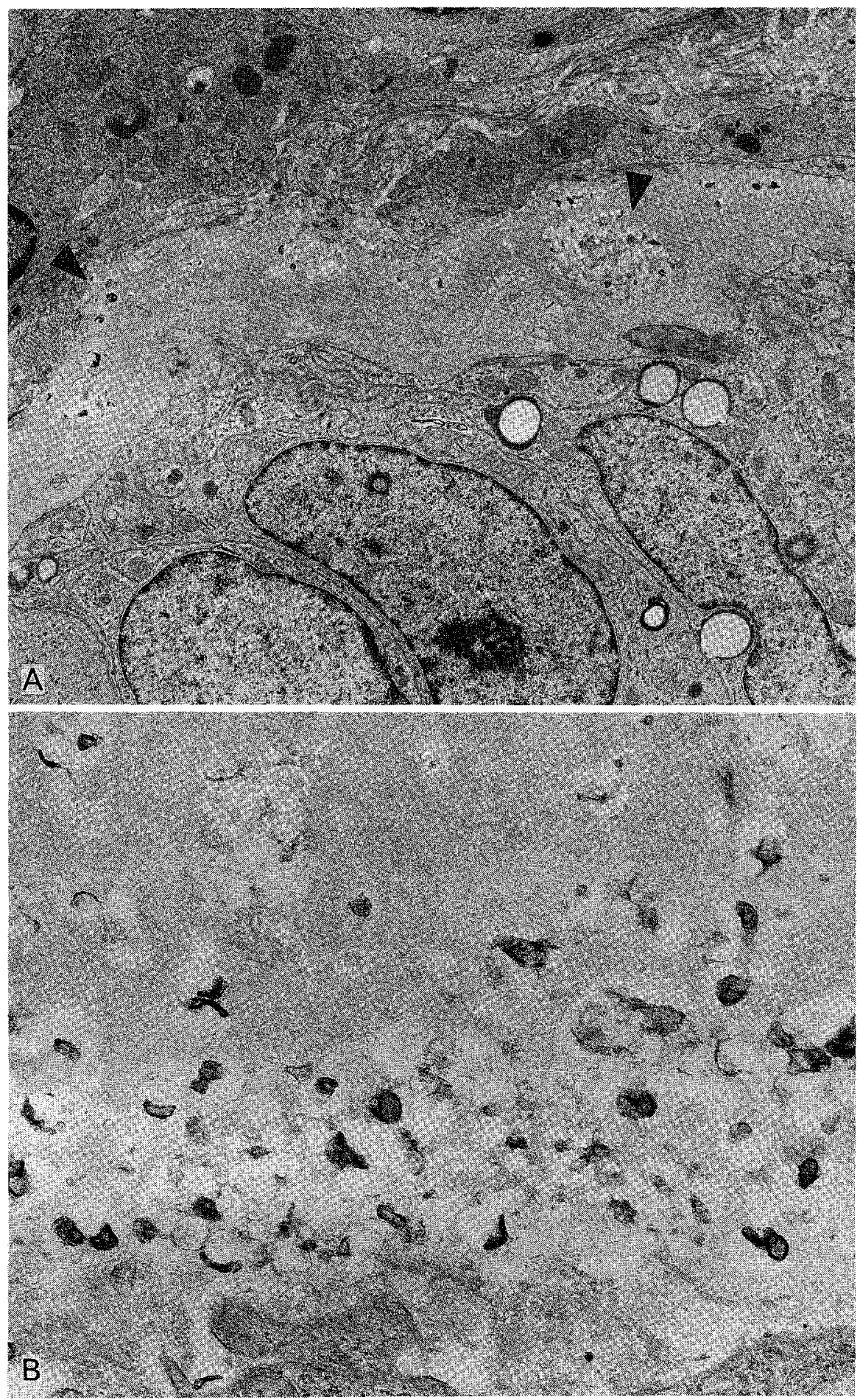

Fig. 6. A) Renal biopsy specimen (electron micrograph). Tubular basement membrane containing numerous electron-lucent areas of rarefaction with osmiophilic particles are present $(\times 9,600)$. Osmiophilic particles are surrounded by electron-lucent areas. B) High magnification of osmiophilic bodies $(\times 36,000)$.

that this patient with IPH displayed evidence of two diseases of immunological origin: chronic thyroiditis and glomerulonephritis.

An association between chronic thyroiditis and IPH is well known in previous reports; Matsuzuka (6), Imai et al (7) and Kato et al (8) noted cases with IPH associated with chronic thyroiditis.

This patient was not considered to have viral hepatitis 
$\mathrm{B}$ as indicated by being negative for HBsAg and having no history of blood transfusion. However, Shikata et al (9) and Umeyama et al (10) reported that HBsAg could be a possible trigger for the induction of IPH.

Czaja's review (11) stated that splenomegaly was present in cases of autoimmune hepatitis without cirrhosis, although infrequent. However, we considered IPH in the present case to be accompanied by immunological reactions, since positive anti-smooth muscle antibody declined after splenectony. The national survey of IPH made by Ohta et al (12) showed an association with immunological abnormalities in IPH patients. Furthermore, autoimmune diseases including chronic thyroiditis were infrequently associated IPH patients. Yoshikawa (13) reported that autoantibodies in patients with IPH disappeared or decreased after splenectomy. They suggested that there are several immunological factors inducing pathogenesis of IPH. The present case also suggested that there is a close connection between IPH and autoimmune thyroiditis.

In the present case with chronic thyroiditis, diffuse mesangial proliferative glomerulonephritis resembles the lesions of IgA nephropathy. Some electron-dense deposits were found sporadically in the mesangial matrix and in the intramembranous areas. O'Regan et al (14) and Mizuno et al (15) reported patients with chronic thyroiditis associated with renal disorders. According to their reports, the renal histological findings showed membranous, membranoproliferative and endocapillary proliferative glomerulonephritis. Deposits of immunoglobulins and thyroglobulin were found in the glomeruli of some of these patients. Calder et al (16) indicated that deposits of thyroglobulin in the glomeruli might play an important role in producing glomerular injury.

Positivity for circulating immune complexes and antithyroglobulin antibody were recognized in the present patient. However, thyroglobulin in glomeruli was negative. We have no definite evidence to indicate that a relationship existed between Hashimoto's disease and the development of glomerulonephritis in our case.

The patient reported here had characteristic renal specimen features showing IgA deposits and no evidence of membranous glomerulonephritis. Sakaguchi et al (3) mentioned three types of deposits in the electron microscopic findings of hepatic glomerulosclerosis, which were expressed as fine osmiophilic granules, amorphous material, and irregular black particles being respectively included in the subendothelial space along the basement membrane or within the basement membrane and in the mesangial matrix. Slight tubular alterations were seen regardless of the severity of glomerular lesions. Callard et al (17) detected glomerular lesions in immune complextype glomerulonephritis in patients with cirrhosis of the liver. These authors pointed out that these lesions consisted of thickening of the basement membrane, electrondense deposits in the mesangium and in capillary walls, and areas of rarefaction in the membrane-like material and in some deposits. They suggested that the deposits in the glomeruli could be either aggregated immunoglobulins or CICs.

We can state clear distinctions on electron micrography between our case and their reports, in which many osmiophilic bodies in the TBM were found with lucent areas of rarefaction and immune dense deposits were seen in the glomerular basement membranes. We consider that lesions of the TBM in this case are as viewed in the electron microscopic study very similar to osmiophilic granular electron dense material detected in bile duct basement membranes of biliary cirrhosis as reported by Bernuau et al (18). However, whether or not origins of $\operatorname{IgA}$ deposits in the present case are related to immunological mechanisms involved with autoimmune thyroiditis or IPH remains to be resolved.

The electron microscopic appearance of this case, characterized by numerous electron-lucent areas of rarefaction with osmiophilic particles in the TBM, suggested that the pathogenesis of the tubulo-interstitial disorder could be related to immunological reaction. We thus consider in our case with dual disorders in renal tissue whether or not interstitial nephritis was induced by the immunological mechanism involved with autoimmune thyroiditis, while this renal involvement could not be definitely qualified as SLE or other autoimmune diseases.

Brentjens et al (19) reported that a concomitant interstitial nephritis frequently develops in SLE nephritis. They stated that that the same immune complexes producing glomerulonephritis are associated with the development of interstitial and tubular lesions. Orfila et al $(20,21)$ and Klassen et al $(22,23)$ described that a distinctive renal tubular lesion was observed characterized by deposition of immunoglobulins along the TBM and by corresponding electro-dense deposits in human renal allografts or SLE and some renal diseases. Furthermore, they mentioned that electron-dense deposits inside or along the TBM seemed to appear specifically in cases of granular deposits. In rare cases, deposits disappeared leaving a lucent space. Lehman et al (24) documented that extraglomerular immunoglobulin occurred in association with glomerular immunoglobulin. MorelMaroger et al (25) and McCluskey and Klassen (26) reported that tubular and interstitial lesions can result from immune complexes or from autoantibodies against tubular basement membrane constituents.

Accordingly, we consider that electron-dense deposits within the TBM are probably due to immune complexes formed locally. The possible explanation for tubulointerstitial nephritis is an immunologic process stimulated by the persistence in the kidney of some antigens which is apt to be caused in an autoimmune disease. Indeed, Rakotoarivony et al (27), and Andres and McCluskey (28) demonstrated the evidence that immune complexes or anti-tubular basement membrane antibody may induce 
tubular and interstitial nephritis in experimental animals.

Although IgA deposits in glomeruli were detected in the present case including autoimmunological disease, IgA nephropathy was not clarified in through strict criteria. Frasca et al (29) reported with patients with IgA nephropathy and tubulo-interstitial lesions representing tubular deposits on immunofluorescence. The deposits consisted of only complement $\mathrm{C3}$, and electrondense deposits were not found at all along the TBM in these patients. These findings are, however, different from tubulo-intestinal lesions in our case.

The present case was positive for the Raji cell test in the low level. However, it can not be evaluated by simply this case whether or not CICs play any pathogenetical role in the development of the renal lesions. Coppo et al (30) and Cairn et al (31) stated that CICs were not linked directly to the renal lesion and the complexity of the immunological mechanisms involved in the pathogenesis of glomerulonephritis does not leave any room for a close correspondence between CICs and the development of disease, although a variety of CICs are found in idiopathic glomerulonephritis. Animal experimental evidence suggested that CIC injection performed only rarely and transiently induced in situ immune complex formation in the subepithelial area (32).

Some investigators have thus recognized that tubulointerstitial nephritis may be produced in experimental animals or in man by immunological mechanisms. We therefore, propose four possibilities for explanation of the pathogenesis of the present case. First, Hashimoto's disease coexists frequently with other autoimmune diseases. However, systemic lupus erythematosus, rheumatoid arthritis, and Sjögren's syndrome could be ruled out in this case. Second, several case reports strongly suggested that autoimmune factors play a prominent role in the association of Hashimoto's disease and IPH. Third, in the same way, autoimmunity in IPH associated with Hashimoto's disease is related to the development of renal lesions including severe interstitial nephritis by immunological mechanisms. Fourth, alternations in the kidneys of this case representing $\operatorname{IgA}$ deposits and severe tubulo-interstitial nephritis are not supported by the findings demonstrated in interstitial nephritis associated with IgA nephritis.

Acknowledgments: The authors wish to thank Associate Professor Takeyuki Kitajima for helpful suggestions.

\section{References}

1) Jordan SC, Johnston WH, Bergstein JM. Immune complex glomerulonephritis mediated by thyroid antigens. Arch Pathol Lab Med 102: 530, 1978.

2) Ploth DW, Fitz A, Schnetzler D, Seidenfeld J, Wilson CB. Thyroglobulin-antithyroglobulin immune complex glomerulonephritis complicating radioiodine therapy. Clin Immunol Immunopathol 9: 327, 1978.
3) Sakaguchi $\mathrm{H}$, Dachs S, Grishman E, Paronetto F, Salomon M, Churg J. Hepatic glomerulosclerosis: An electron microscopic study of renal biopsies in liver diseases. Lab Invest 14: 533, 1965.

4) Tokuda T. A morphological study on Banti livers, with special reference to their angioarchitecture. Jikeikai Med J 92: 399, 1977 (in Japanese; abstract in English).

5) Yamamoto K. Morphological studies of the spleen in idiopathic portal hypertension (so-called Banti's syndrome without liver cirrhosis) using light microscopy, scanning electron microscopy and histometry. Acta Pathol Jpn 29: 1, 1979.

6) Matsuzuka F. Relation of chronic thyroiditis to hepatic and splenic disorders, with special reference to Banti's syndrome. Fukushima J Med Sci 26: 21, 1976 (in Japanese; abstract in English).

7) Imai $Y$, Minami $Y$, Miyoshi S, et al. Idiopathic portal hypertension associated with Hashimoto's disease: report of three cases. Am J Gastroenterol 81: 791, 1986.

8) Kato S, Asakura H, Ichikawa E, Kuramochi S, Tsuzuki T, Tsuchiya M. A case of idiopathic portal hypertension with Cruveilhier-Baumgarten syndrome, complicated with nodular hyperplasia of the liver and chronic thyroiditis. Jpn J Gastroenterol 84: 1314, 1987 (in Japanese; abstract in English).

9) Shikata T, Uzawa T, Yamazaki S. Causalgenesis of liver fibrosis accompanied with portal hypertension. Acta Hepatol Jpn 17: 417, 1976 (in Japanese; abstract in English).

10) Umeyama K, Yui S, Fukamizu A, Yoshikawa K, Yamashita T. Idiopathic portal hypertension associated with progressive systemic sclerosis. Am J Gastroenterol 77: 645, 1982.

11) Czaja AJ. Natural history, clinical features and treatment of autoimmune hepatitis. Semin Liver Dis 4: 1, 1984.

12) Ohta G, Saito K, Nakanuma Y. A Survey of Idiopathic Portal Hypertension (IPH) Associated with Autoimmune Phenomena. In: 1983 Report of the Ministry of Health and Welfare Research Committee on Idiopathic Portal Hypertension. Okuda K, Ed. Tokyo, 1984, p. 176 (in Japanese; abstract in English).

13) Yoshikawa K. Immunological studies in the etiology of idiopathic portal hypertension. J Jpn Soc Surg 79: 538, 1978 (in Japanese; abstract in English).

14) O'Regan S, Fong JSC, Kaplan BS, Chadarévian JPD, Lapointe $\mathrm{N}$, Drummond KN. Thyroid antigen-antibody nephritis. Clin Immunol Immunopathol 6: 341, 1976.

15) Mizuno $M$, Hasegawa $H$, Fujishiro $T$, et al. Chronic thyroiditis complicated by nephrotic syndrome and marked hydroureteronephrosis. Jpn J Nephrol 29: 561, 1987.

16) Calder EA, Penhale WJ, Barnes EW, Irvine WJ. Evidence for circulating immune complex in thyroid disease. Br Med $\mathrm{J}$ 2: 30,1974 .

17) Callard P, Feldmann G, Prandi D, et al. Immune complex type glomerulonephritis in cirrhosis of the liver. Am J Pathol 80: $329,1975$.

18) Bernuau D, Feldmann G, Degott C, Gisselbrecht C. Ultrastructural lesions of bile ducts in primary biliary cirrhosis. A com. parison with the lesions observed in graft versus host disease. Human Pathol 12: 782, 1981.

19) Brentjens JR, Sepulveda M, Baliah $T$, et al. Interstitial immune complex nephritis in patients with systemic lupus erythematosus, Kidney Int 7: 342, 1975.

20) Orfila C, Rakotoarivony I, Durand D, Suc J-M. A correlative study of immunofluorescence, electron and light microscopy in immunologically mediated renal tubular disease in man. Nephron 23: 14, 1979.

21) Orfila C, Vega-Vidallé C, Suc JM. Ultrastructural changes of tubular basement membranes in immunologic renal tubular lesions in humans. Ultrastruct Pathol 14: 121, 1990.

22) Klassen J, Kano K, Milgrom F, et al. Tubular lesions produced by autoantibodies to tubular basement membrane in human renal allografts. Int Arch Allergy 45: 675, 1973. 


\section{Sasaki et al}

23) Klassen J, Andres GA, Brennan JC, McCluskey RT. An immunologic renal tubular lesion in man. Clin Immunol Immunopathol 1: $69,1972$.

24) Lehman DH, Wilson CB, Dixon FJ. Extraglomerular immunoglobulin deposits in human nephritis. Am J Med 58: 765, 1975.

25) Morel-Maroger L, Kourilsky O, Mignon F, Richet G. Antitubular basement membrane antibodies in rapidly progressive poststreptococcal glomerulonephritis: Report of a case. Clin Immunol Immunopathol 2: 185, 1974.

26) McCluskey RT, Klassen J. Immunologically mediated glomerular, tubular and interstitial renal disease. $\mathrm{N}$ Engl J Med 288: 567, 1973.

27) Rakotoarivony J, Orfila C, Segonds A, et al. Human and experimental nephropathies associated with antibodies to tubular basement membrane. Adv Nephrol 10: 187, 1981.

28) Andres GA, McCluskey RT. Tubular and interstitial renal diseases due to immunologic mechanisms. Kidney Int 7: 271, 1975.

29) Frasca GM, Vangelista A, Biagini G, Bonomini V. Immunological tubulo-interstitial deposits in IgA nephropathy. Kidney Int 22: 184, 1982.

30) Coppo R, Roccatello D, Basolo B, Piccoli G. Circulating immune complexes in immunologically mediated glomerular diseases. Contr Nephrol 48: 137, 1985.

31) Cairn S, London A, Mallick NP. Circulating immune complexes in idiopathic glomerular disease. Kidney Int 21: 507, 1982.

32) Couser WG, Salant DJ. In situ immune complex formation and glomerular injury. Kidney Int 17: 1, 1980. 